\title{
Nonlinear Vibration Analysis of Machine-Tool Guideway System with Clearance
}

\author{
Haitao Luo ${ }^{1,2, ~ a, ~ Y u w a n g ~ L i u, ~ 2, ~ b, ~ H o n g g u a n g ~ W a n g ~}{ }^{1}$, Weijia Zhou ${ }^{1}$ \\ ${ }^{1}$ State Key Laboratory of Robotics, Shenyang Institute of Automation \\ Chinese Academy of Sciences, Shenyang 110016, China \\ ${ }^{2}$ Graduate School of the Chinese Academy of Sciences, Beijing 100039, China \\ aluohaitao@sia.cn, bliuyuwang@sia.cn
}

\section{Keywords: Nonlinear vibration, Hysteretic effect, Double-period, Chaos}

\begin{abstract}
This paper studies hysteretic effect of machine-tool guideway system with clearance, establishes the nonlinear dynamics model of guideway system with symmetric clearance, and obtains the equivalent damping and equivalent stiffness coefficients using asymptotic method. With the use of time-domain graph, frequency-domain graph, phase trajectory figure, Poincare section figure, bifurcation diagram, and lyapunov exponent figure, the dynamics response of damping and external vibration frequency effect on system is studied. The result shows that the period, double-period and chaos movement will occur when the system parameters change in certain range.
\end{abstract}

\section{Introduction}

Machine-tools are composed of many parts according to certain requirements. We usually call the contact region joint where some parts contact mutually and load transferred each other. While in the modern machine structural design process, the guideway system is the common joint type. Under the effect of dynamics loads, the guideway joint always shows both flexibility and damping characteristics. And, due to the existence of clearance, hysteretic curve will be formed during the vibration [1]. Hysteretic system is a kind of typical dissipative system which posses the bifurcation and singularity characteristics. With the change of related parameters, system will appear extremely complex nonlinear vibration. It seriously affects the machining accuracy of machine-tool.

According to the engineering application, hysteretic system can be divided into two categories: piecewise linear hysteretic model and curve hysteretic model. Caughy firstly put forward a kind of double linear piecewise linear hysteretic model in 1960. This model has symmetric property, and the force-deformation curve is composed of several different lines. Thereafter based on this theory, Neilsen and Clough propose a variety of degenerated double-line and linear hysteretic model. Then Bouc puts forward a smooth curve hysteretic model, which is controlled by differential equation. Until now, this model is still widely used [2,3]. Later, Wen ect improve this model and prove a series of different hysteretic curves [4]. Due to the existence of clearance between guideway and guideblock, machine-tool guideway system is a typical double-linear piecewise linear hysteretic model.

This paper firstly presents the dynamics model and creates the nonlinear vibration equation of machine-tool guideway system. Thereafter we compute the equivalent damping and equivalent stiffness coefficients using asymptotic method. These works provides theoretical basis for the next research. Finally, with the use of time-domain, frequency-domain, phase trajectory, poincare section, bifurcation diagram, and lyapunov exponent analysis tools, the dynamics response of damping and external vibration frequency effect on system is studied. Through the dynamics characteristics analysis on guideway hysteretic system with symmetric clearance, it can help us improve the machining precision and dynamic performance. In addition, it also laid a solid theoretical foundation for the next vibration control and suppression. 


\section{Hysteretic Model of Guideway System with Clearance}

The joint of machine-tool guideway system belongs to flexible junction. Generally speaking, the joint surface is larger and pressure distribution is no uniform, so we should choose some points to simulate different contact state on different joint surface. On the contrary, if the joint surface is smaller, pressure distribution is relative uniform and the contact state on joint surface is basic agreement, we can use only a combining point instead of joint surface.

We assume that the interactive property of guideway joint suffered on periodic external excitation is uniform in full pressure direction; the elastic-plastic deformation is also consistent in the two directions, which exists symmetric hysteretic force. On the premise of not considering the horizontal friction, namely the guideway joint is only under the force in normal direction. We establish the single freedom model of machine-tool guideway joint, as shown in Fig.1.
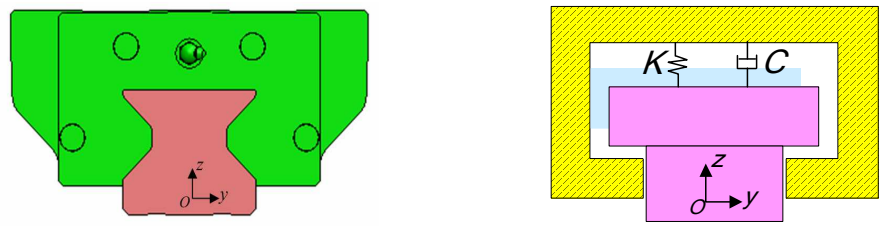

Fig. 1. Single degree of freedom model of guideway joint

Due to the existence of clearance on guideway joint, the real force conditions would be played a role in the two directions. Assume that the guideway of machine-tool is stationary; there exist symmetric clearance between slide-way and slide-block; considering the action of contact stiffness and contact damping, the slide-block makes vertical vibration along z-axis. In this case, we deeply analyze the guideway joint of machine-tool and establish the mathematical model of the system, as shown in Fig.2.

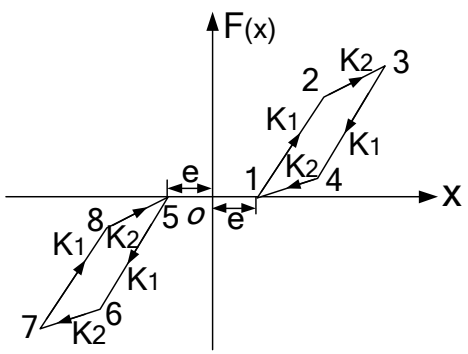

Fig. 2. Piecewise linear hysteretic model with symmetric clearance

Because the system has the characteristics of hysteretic model, the motion differential equations can be expressed as:

$$
m \ddot{x}+c \dot{x}+F(x)=P \sin \omega t,
$$

where, $m-$ mass of system;

$c$ - damping of system;

$P$ _ amplitude of external excitation force;

$F(x)$ double linear hysteretic force.

$$
F(x)=\left\{\begin{array}{cl}
0 & |x| \leq e \\
k_{1} x+\alpha_{1} \operatorname{sgn}(x) & e \leq|x| \leq x_{2}, x \dot{x}>0 \\
k_{2} x+\alpha_{2} \operatorname{sgn}(x) & x_{2} \leq|x| \leq x_{3}, x \dot{x}>0, \\
k_{1} x+\alpha_{3} \operatorname{sgn}(x) & x_{4} \leq|x| \leq x_{3}, x \dot{x}<0 \\
k_{2} x+\alpha_{4} \operatorname{sgn}(x) & e \leq|x| \leq x_{4}, x \dot{x}<0
\end{array}\right.
$$


where, $k_{1} \_$loading stiffness in the elastic stage;

$k_{2} \_$loading stiffness in the plastic stage;

$e-$ clearance;

$F(x) —$ double linear hysteretic force.

Here, $\alpha_{1}=-k_{1} e, \alpha_{2}=-k_{2} x_{2}+F_{2}, \alpha_{3}=-k_{2} x_{3}+F_{3}, \alpha_{4}=-k_{2} e, F_{2}=k_{1}\left(x_{2}-e\right), F_{3}=F_{2}+k_{2}\left(x_{3}-x_{2}\right)$.

\section{Asymptotic Solution of System Equation}

Asymptotic method, also named three series method or KBM method. It was put forward by Krylov, Poglupov and Mitropolsci of former Russian scholars [1]. This method and the general method is not quite the same. It refers the equation solutions and vibration amplitude as to power series of small parameter $\varepsilon$. And then, we can obtain these unknown coefficients of power series using the method of variables separation.

The quantitative analysis for nonlinear system with hysteretic restoring force is an important problem in engineering. In the use of asymptotic method for the concrete solution, the system can be divided into several linear segments. So we can solve these piecewise linear nonlinear system by subsection integration method.

Assume that the approximate solution of nonlinear system is $x=a \sin \theta$. When $x=e$, the corresponding phases are $\varphi_{1}=\arcsin (e / a), \varphi_{2}=\arcsin \left(x_{2} / a\right), \varphi_{3}=\arcsin \left(x_{3} / a\right)$ and $\varphi_{4}=\arcsin \left(x_{4} / a\right)$, the first derivative of amplitude and phase relative to time can be expressed as:

$$
\left\{\begin{array}{l}
\dot{a}=\varepsilon A_{1}(a) a \\
\dot{\theta}=\omega_{0}+\varepsilon B_{1}(a)
\end{array},\right.
$$

Here, $\omega_{0}=\sqrt{k / m}$, for other parameters, see reference documents[5]. Introducing formula $x_{0}=a \sin \varphi$ and $\dot{x}_{0}=a \omega_{0} \cos \varphi$, through the asymptotic method, we can deduce that the equation is as follow:

$$
\left\{\begin{array}{l}
A_{1}(a)=-\frac{1}{2 \pi \omega_{0}} \int_{0}^{2 \pi} f_{0}(a, \varphi) \cos \varphi d \varphi \\
B_{1}(a)=\frac{1}{2 \pi \omega_{0} a} \int_{0}^{2 \pi} f_{0}(a, \varphi) \sin \varphi d \varphi
\end{array} .\right.
$$

Putting relative numerical value into equations and organizing it, we can obtain the equivalent stiffness and equivalent damping of system. The concrete coefficients are as follow:

$$
\begin{aligned}
k_{e}= & \frac{k_{1}}{\pi}\left[\frac{1}{2}\left(\varphi_{1}-\varphi_{2}+\varphi_{4}\right)+\frac{1}{4}\left(\sin 2 \varphi_{1}-\sin 2 \varphi_{2}+\sin 2 \varphi_{4}\right)\right] \\
- & \frac{k_{2}}{\pi}\left[\frac{1}{2}\left(\varphi_{1}+\varphi_{2}-\varphi_{4}\right)+\frac{1}{4}\left(\sin 2 \varphi_{1}-\sin 2 \varphi_{2}-\sin 2 \varphi_{4}\right)\right], \\
+ & \frac{k_{2} e}{\pi a}\left(\sin \varphi_{4}-\sin \varphi_{1}\right) \\
c_{e}= & -\frac{1}{\pi a \omega}\left[a_{1}\left(\cos \varphi_{1}-\cos \varphi_{2}\right)+a_{2}\left(\cos \varphi_{2}-1\right)\right]+\alpha_{3}\left(1-\cos \varphi_{4}\right) \\
& +\alpha_{4}\left(\cos \varphi_{4}-\cos \varphi_{1}\right)+\frac{k_{1} a}{4}\left(\cos 2 \varphi_{1}-\cos 2 \varphi_{2}\right) \\
& +\frac{k_{2} a}{4}\left(\cos 2 \varphi_{2}-\cos 2 \varphi_{1}+\cos 2 \varphi_{4}-1\right)
\end{aligned}
$$




\section{Numerical Solution of System Equation}

Because the guideway joint of machine-tool has the vibration characteristics of hysteretic system, we commonly use numerical method to analyze the vibration characteristics of hysteretic system. These methods mainly include today popular time-domain, frequency-domain, phase trajectory, poincare section, bifurcation diagram, and lyapunov exponent analysis tools [6-10]. Generally speaking, for the solution of piecewise linear hysteretic vibration system, the whole calculation time should be divided into many small time steps and the restoring force needed to be defined as constant. That is to say, we make the approximate solution with piecewise linear method.

For the convenience of analysis, we rewrite the differential Eq. 1 as:

$$
\ddot{x}+2 \xi \omega \dot{x}+f(x)=P \sin \omega t,
$$

Here, $p=P / m$, nonlinear hysteretic force is as follow.

$$
F(x)=\left\{\begin{array}{cl}
0 & |x| \leq e \\
\omega_{1}^{2} x+\alpha_{1} \operatorname{sgn}(x) & e \leq|x| \leq x_{2}, x \dot{x}>0 \\
\omega_{2}^{2} x+\alpha_{2} \operatorname{sgn}(x) & x_{2} \leq|x| \leq x_{3}, x \dot{x}>0, \\
\omega_{1}^{2} x+\alpha_{3} \operatorname{sgn}(x) & x_{4} \leq|x| \leq x_{3}, x \dot{x}<0 \\
\omega_{2}^{2} x+\alpha_{4} \operatorname{sgn}(x) & e \leq|x| \leq x_{4}, x \dot{x}<0
\end{array}\right.
$$

Here, $\omega_{1}^{2}=k_{1} / m, \omega_{2}^{2}=k_{2} / m, \alpha_{1}=-\omega_{1}^{2} e, \alpha_{2}=-\omega_{2}^{2} x_{2}+f_{2}, \alpha_{3}=-\omega_{1}^{2} x_{3}+f_{3}, \alpha_{4}=-\omega_{2}^{2} e$, $f_{2}=\omega_{1}^{2}\left(x_{2}-e\right), f_{3}=f_{2}+\omega_{2}^{2}\left(x_{3}-x_{2}\right)$.

Response of external excitation frequency on system. In the actual machining process of machine-tool, the vibration will happen due to contact and friction between end cutting-tool and work-piece. The size and speed of vibration amplitude depend on the stiffness and inherent frequency of this system. We usually take the introduction force due to vibration as to external excitation force. When the frequency of end excitation force is equal or approach to the inherent frequency of guideway system, the resonance will occur. This will greatly influence the machining precision of machine-tool. So it is very important to analyze the response of external excitation frequency relative to guideway system.

Here, we set the parameters of system equation are $e=0.2 \mu \mathrm{m}, x_{2}=2 \mu \mathrm{m}, x_{3}=3.5 \mu \mathrm{m}$ and $x_{4}=2 \mu \mathrm{m}$. In addition, in order to solve the response of external excitation frequency act on system, we also assume that system damping is $2 \xi \omega=0.46, p=3.5, \omega_{1}=560$ and $\omega_{2}=380$. According to the characteristic parameters' changes of system, we can get the time-domain, frequency-domain, phase trajectory, poincare section figure, as shown in Fig.3.

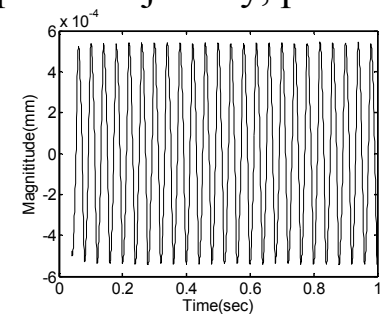

(a) Time-domain figure

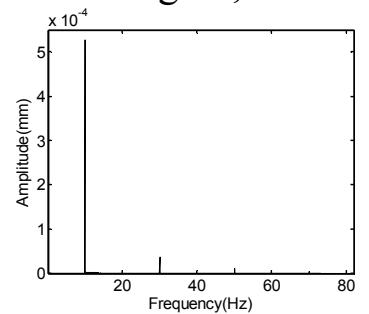

(b) Frequency-domain figure

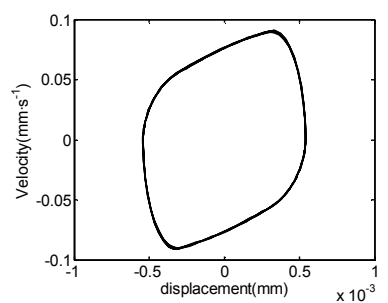

(c) Phase trajectory figure

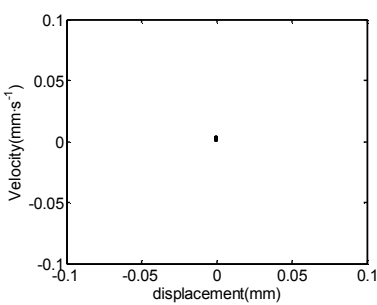

(d) Poincare section figure

Fig. 3. Response curve of system under the $f=10 \mathrm{~Hz}$.

From the Fig.3, we can see that the whole system is always in periodical motion state under the external excitation frequency $f=10 \mathrm{~Hz}$. The time-domain waveform is neat, frequency characteristic curve appear times frequency phenomenon, the phase trajectory is the single cycle close curve, and the poincare section is a point. All of these features indicate that the system is in periodic movement. 


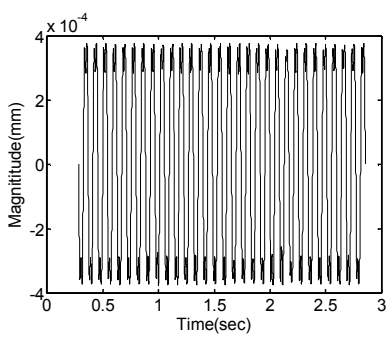

(a) Time-domain figure

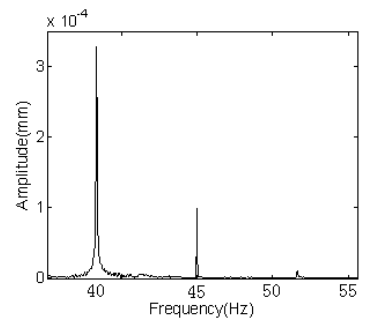

(b) Frequency-domain figure

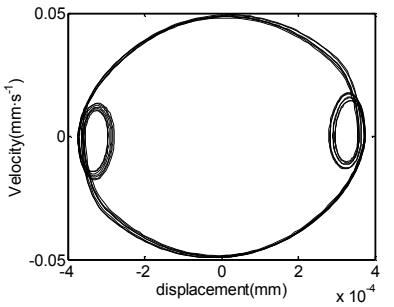

(c) Phase trajectory figure

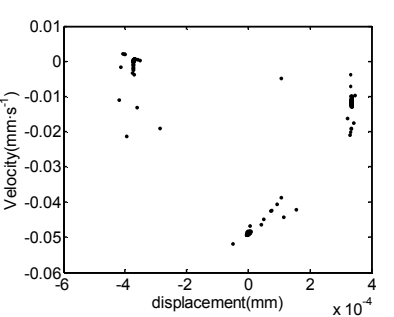

(d) Poincare section figure

Fig. 4. Response curve of system under the $f=40 \mathrm{~Hz}$.

From the Fig.4 above, we can see that the whole system is always in multiple periodic movement state under the external excitation frequency $f=40 \mathrm{~Hz}$. The time-domain waveform appear peak-cutting phenomenon, times frequency phenomenon of frequency characteristic curve is remarkable, the phase trajectory figure appears three rings close curve, and the poincare section is a series of points in three separate areas. All of these features indicate that the system is in double periodic motion.

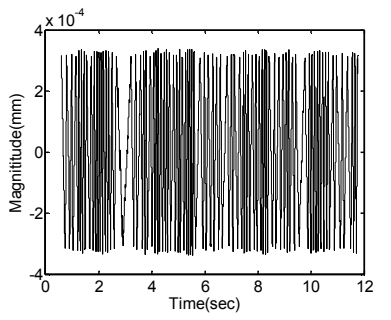

(a) Time-domain figure
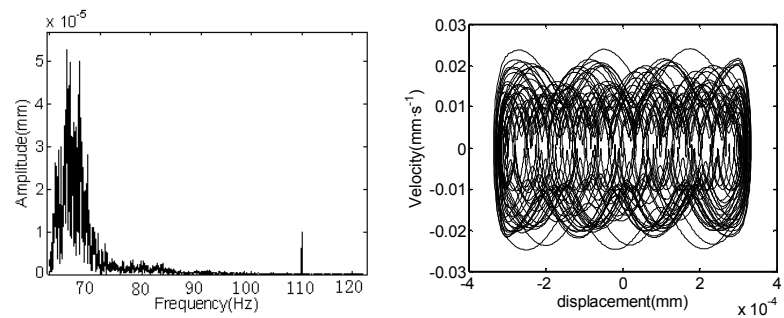

(b) Frequency-domain figure (c) Phase trajectory figure (d) Poincare section figure

Fig. 5. Response curve of system under the $f=70 \mathrm{~Hz}$.

From the Fig.5 above, we can see that the whole system appears chaos movement under the external excitation frequency $f=70 \mathrm{~Hz}$. The time-domain waveform is lack of regularity, the scope of frequency characteristic curve is wider, the phase trajectory figure is in a mess, and the points on the poincare section are irregular. All of these features indicate that system appears chaos movement.

Response of damping on system. In the machine-tool operation process, the damping property of joint has an effect on dynamics performance. With contact vibration, the damping value of joint will increase. It is an important cause of inherent frequency fall. Generally, the damping value caused by contact vibration is often larger than the parts themselves, and this kind of effect is obvious. In order to study the machine guideway joint system, if the nonlinear hysteretic force can cause the chaos phenomenon, we assume that the restoring forces fully undertake by nonlinear hysteretic forces.

In the same, we assume that the coefficients of system equations are $e=0.2 \mu \mathrm{m}, x_{2}=2 \mu \mathrm{m}, x_{3}=3.5 \mu \mathrm{m}$ and $x_{4}=2 \mu \mathrm{m}$. In addition, in order to solve the response of system damping act on system, we also assume that the other coefficients are $f=80, p=3.5, \omega_{1}=560$ and $\omega_{2}=380$. According to the characteristic parameters' changes of system, we may get the bifurcation diagram, and lyapunov exponent figure, as shown in Fig.6.

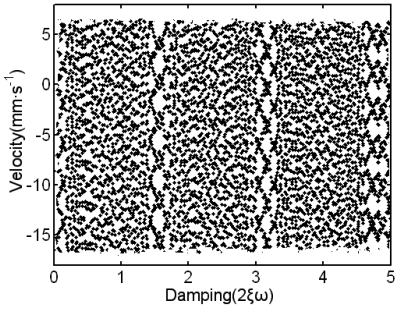

(a) bifurcation diagram

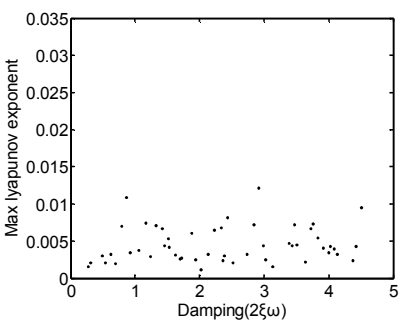

(b) Max Lyapunov exponent

Fig. 6. Bifurcation diagram and lyapunov exponent figure 
From the Fig. 6 above, we can see that the guideway system is always in chaos state. The max lyapunov exponents are all grater than zero. Guideway hysteretic system with symmetric clearance will consume system's energy because of the existence of restoring forces. When the damping is negative, for example the self-excited vibration, the system is easier to appear chaos movement behavior. While mechanical systems contain hysteretic system's vibration characteristics, when the damping value is positive, the whole system also appears chaos movement.

\section{Conclusion}

This paper mainly analyzed hysteretic effect of machine-tool guideway system with symmetric clearance, studied system's nonlinear dynamics behavior and importantly discussed the dynamics response with damping and external vibration frequency changes. The conclusion is as follows:

1) With the frequency change of external excitation force, the system occurs the period, double-period and chaos movement. Meanwhile, the time-domain waveform begins to appear peak-cutting phenomenon; the frequency scope is gradually wider; the phase trajectory figure appears from single cycle to multiple cycles' movement and the poincare section is more and more irregular.

2) With the damping parameter change of joint, the guideway system is always in chaos movement state. From the graph, we can see that the bifurcation diagram is in a mess and the max lyapunov exponents are all grater than zero. These phenomenons proved the chaos movement occurs.

3) Through the dynamics characteristics analysis on guideway hysteretic system, it can help us further improve the machining precision and enhance the dynamic performance of the whole machine. In addition, it also creates the conditions for the next vibration control and suppression.

\section{Acknowledgment}

This work was supported by the National Science and Technology Major Project on High Grade CNC Machine Tool and Fundamental Manufacturing Equipment under Grant 2010ZX04007-011. Special thanks to Yuwang Liu, Yuanzheng Tian and Minhong Wan who have offered a lot of contribution to the paper.

\section{References}

[1] Bangchun Wen, Yinong Li, Peimin Xu and Qingkai Han, Engineering nonlinear vibration, First edition, Science Press, Beijing, 2007.

[2] Jiahao Lin, Naisi Qu, Huancun Shun, Computational Dynamics of Structure, First edition, University of Technology Press, Dalian, 1989.

[3] Caughey T K, Random excitation of a system with bilinear hysteresis, J. ASME Journal of Applied Mechanics. 12(1960) 649-652.

[4] Wen Y K, Method for random vibration of hysteretic systems, J. Proceedings of ASCE, Journal of Engineering Mechanics. 12(1976) 249-263.

[5] Hongguang Li, Nonlinear Dynamic Analysis of Mechanical System with Clearance and Hysteretic Characteristics, J. Shanghai Jiao tong University, 2001.

[6] Wenge Zhang, Yu wang and Shijun Wang, Study on Bifurcations and Chaos of Slideway-Joint System, J. Noise and Vibration Control, 43(1996) 43-47.

[7] Hongguang Li, Guang Meng, Double nonlinear vibration of hysteretic system with clearance, J. Chinese Journal of Mechanical Engineering, 40(2004) 10-13.

[8] YinShan Li, Xinye Li, Bo Liu, Bifurcation, Chaos, Nonlinear Oscillations and Their Application in Engineering, J. Journal of Hebei University of Technology. 33(2004) 96-103.

[9] Haiyan $\mathrm{Hu}$, Nosmooth Analysis of Dynamics of a piecewise Linear System. Acta Mechanica Sinica. 28(1996) 483-488.

[10] Guanwei Luo, Jianhua Xie, Researches on Stability of Periodic Motion, Bifurcations and Chaos in A Vibratory System with A Clearance or Gap, J. Acta Mechanica Solida Sinica. 24(2003) 285-292. 